\title{
Promouvoir l'équité entre les genres en médecine
}

\author{
Andrea C. Tricco PhD, Ivy Bourgeault PhD, Ainsley Moore MSc MD, Eva Grunfeld MD PhD, Nazia Peer MD LLM, \\ Sharon E. Straus MD
}

Citation : CMAJ 2021 February 16;193:E244-50. doi : 10.1503/cmaj.200951-f

Voir la version anglaise de l'article ici : www.cmaj.ca/lookup/doi/10.1503/cmaj.200951

$\mathbf{L}$ a profession médicale présente un problème d'iniquité, particulièrement en ce qui concerne le leadership. Plusieurs études canadiennes ont souligné l'ampleur des iniquités sur le plan du leadership en médecine, et particulièrement en ce qui concerne les possibilités d'avancement en milieu universitaire selon le genre. Le genre (défini dans l'encadré 1), de même que la race, la religion, l'orientation sexuelle et l'origine culturelle, suscitent la discrimination, y compris en médecine ${ }^{1}$. Dans cette profession, les iniquités touchant la rémunération et l'avancement professionnel, ainsi que les attitudes discriminatoires de collègues et de patients, ont été associées à un entrecroisement entre race et genre. Or, au fil des ans, la recherche dans ce domaine a rarement tenu compte de l'intersectionnalité.

L'équité entre les genres et la diversité raciale en médecine peuvent engendrer des solutions créatives à des problèmes de santé complexes et favoriser une optimisation des soins. Nous abordons ici le problème de l'iniquité entre les genres dans la profession médicale au Canada, ses causes premières, les enjeux soulevés par l'iniquité pour la profession, et les approches multidimensionnelles pour promouvoir l'équité à tous les niveaux des organisations médicales, selon les meilleures données probantes disponibles, résumées dans l'encadré 2.

\section{Quelle est l'ampleur de l'iniquité entre les genres au sein du leadership médical canadien?}

Même si depuis un quart de siècle, les femmes se font plus nombreuses que les hommes dans les facultés de médecine canadiennes, elles ne sont pas également représentées dans les postes de responsabilité ${ }^{2}$ et sont moins susceptibles que leurs collègues masculins de gravir les échelons supérieurs, même en tenant compte de facteurs tels que l'âge, l'expérience, la productivité et la spécialité ${ }^{3}$. Dans son rapport de 2018, l'Association médicale canadienne (AMC) reconnaissait que l'iniquité entre les sexes chez les leaders médicaux est un

\section{POINTS CLÉS}

- L'iniquité entre les genres persiste en médecine et dans les milieux universitaires médicaux au Canada, particulièrement dans la haute hiérarchie.

- Il a été démontré que l'équité entre les genres va de pair avec une amélioration des politiques en santé et des soins aux patients.

- Des approches multidimensionnelles et fondées sur des données probantes s'imposent pour promouvoir l'équité entre les genres en médecine, à tous les échelons des organisations médicales et à toutes les étapes de la carrière médicale.

problème important ${ }^{4}$. Seulement 8 des 152 anciens présidents de l'AMC ont été des femmes. La première doyenne d'une faculté de médecine canadienne a été nommée en 1999, soit 170 ans après la création de la première faculté de médecine $\mathrm{e}^{5}$, et seulement 8 femmes ont accédé au poste de doyenne depuis ce temps. Yu et ses collègues ont analysé des données de l'Association of American Medical Colleges sur la composition des facultés de médecine aux États-Unis de 1997 à 2008 et ont montré qu'en présence d'intersectionnalité avec race et ethnicité, le fossé entre les genres s'élargit encore davantage. Par exemple, parmi les responsables en médecine interne, 12 étaient des hommes asiatiques, 10 étaient noirs ( 9 hommes et 1 femme), 7 étaient hispaniques ( 5 hommes, 2 femmes) et 137 étaient blancs (116 hommes, 21 femmes). Il faut aussi noter que parmi les membres facultaires, seulement $11 \%$, $9 \%, 11 \%$ et $24 \%$ des femmes asiatiques, noires, hispaniques et blanches respectivement étaient professeures titulaires, contre $21 \%, 18 \%, 19 \%$ et $36 \%$ des hommes asiatiques, noirs, hispaniques et blancs, respectivement ${ }^{6}$.

Plusieurs études ont documenté l'ampleur de l'iniquité entre les genres dans le monde médical universitaire, où la réussite dépend de la capacité d'obtenir des subventions et du nombre de publications et de stagiaires mentorés. Le fossé entre les genres est manifeste dans les concours de bourses de recherche nationaux, tant pour ce qui est des chercheurs ${ }^{7}$, 


\section{Encadré 1 : Multidimensionnalité des genres}

- Selon l'Organisation mondiale de la santé, le genre est un concept multidimensionnel qui reflète les « rôles, comportements, activités, attributs et possibilités que toute société considère appropriée pour les garçons et les filles, les femmes et les hommes $»^{1}$. Le genre n'est pas binaire; c'est un phénomène multidimensionnel. L'iniquité entre les genres s'appuie largement sur les normes, les relations et les rôles genrés. Par contre, la littérature médicale décrit le genre sous une forme binaire (femmes et hommes) et à ce titre, la recherche s'intéresse surtout aux différences entre les femmes et les hommes plutôt que de tenir compte de l'intersectionnalité.

- Les rôles genrés sont façonnés dès la petite enfance et ont une influence sur tous les aspects du développement humain et de la perception du monde (p. ex., attentes traditionnelles envers les femmes pour qu'elles soient des ménagères attentionnées et envers les hommes pour qu'ils soient des pourvoyeurs). Les rôles genrés traditionnels expliquent pourquoi les femmes s'acquittent de tâches non rémunérées plus que les hommes, à la maison et au travail, ce qui affecte leur rendement professionnel.

- Les normes genrées sont des notions répandues et partagées sur la façon dont les gens devraient s'exprimer, se vêtir, se présenter et se comporter en contexte social, professionnel ou privé (p. ex., la confiance en soi est perçue comme un attribut de leadership masculin et on attend des femmes qu'elles soient soumises, ce qui peut mener à de la discrimination ou à des inégalités dans les possibilités offertes selon le genre).

- Les relations genrées font référence à la dynamique qui prévaut entre les genres et qui est déterminée par plusieurs facteurs (p. ex., religion, culture ou société); cette dynamique peut être à l'origine d'iniquités en termes de pouvoir et d'accessibilité ou de contrôle des ressources. Ces iniquités peuvent faire en sorte que les hommes subiront moins de conséquences d'incivilités, ou se croiront autorisés plus que d'autres genres à faire subir du harcèlement.

\section{Encadré 2 : Données utilisées pour le présent article}

Nous avons interrogé les réseaux PubMed et MEDLINE pour recenser les articles de langue anglaise publiés à n'importe quel moment en date d'août 2020, à partir des mots clés "solutions", " gender inequity » et « medicine ». Nous avons inclus des essais randomisés et contrôlés, des revues systématiques, des méta-analyses et des études d'observation. Nous avons aussi consulté la littérature grise sur Google et Google Scholar, les bibliographies et listes de référence des articles retenus, le dossier du projet du groupe Gender in Global Research établi par Elsevier et divers sites Web canadiens et internationaux, notamment l'Association of American Medical Colleges, l'Association des facultés de médecine du Canada, la Society for Canadian Women in Science and Technology, l'American Medical Women's Association et l'Institut de la santé des femmes et des hommes des Instituts de recherche en santé du Canada. que des projets 8 . Si plus de chercheurs que de chercheuses décrochent des subventions après qu'on ait tenu compte de facteurs tels que l'âge et l'expérience, cela exacerbe encore plus les disparités et affecte négativement la trajectoire professionnelle des femmes. Par exemple, plus une personne obtient de subventions, plus elle attirera de stagiaires et plus sa réussite et sa productivité augmenteront, pavant ainsi la voie à des promotions et éventuellement à un poste de professeur titulaire. Les lignes directrices de pratique clinique sont largement utilisées pour orienter la pratique et elles sont souvent citées, or les femmes médecins sont sousreprésentées dans les comités de rédaction des lignes directrices et sont moins susceptibles que les hommes d'en être les auteures principales ${ }^{9}$. Les séances scientifiques sont des occasions de former les leaders et de promouvoir la diversité en médecine; mais selon une étude rétrospective de 2018 sur les présentateurs des séances scientifiques médicales dans 5 grands centres hospitaliers universitaires canadiens, les femmes sont sous-représentées ${ }^{10}$. Comparativement aux hommes, les femmes sont plus susceptibles de travailler dans des secteurs de la médecine moins bien rémunérés et généralement moins valorisés ${ }^{11,12}$, d'obtenir des lettres de recommandation moins élogieuses que leurs collègues masculins pour des postes dans les facultés de médecine ${ }^{13}$ et d'être moins souvent interpelées par leur titre professionnel ${ }^{14,15}$. En milieu universitaire comme dans la pratique, les femmes sont moins bien rémunérées que les hommes, même après la prise en compte de plusieurs facteurs tels que l'âge, l'expérience et la charge de travail11,12; en fait, selon des estimations, le revenu des femmes serait en moyenne de $30 \%$ et $40 \%$ inférieur à celui des hommes selon le modèle de rémunération à l'acte pour les médecins de famille et les spécialistes, respectivement.

\section{Quels facteurs contribuent à cette iniquité entre les genres?}

Le problème d'iniquité entre les genres dans le leadership médical n'a rien à voir avec un quelconque manque de candidates ayant l'expérience et la formation voulues pour assumer des rôles de responsabilité; il ne s'explique pas non plus par une simple soi-disant différence entre les aspirations des unes et des autres ${ }^{16}$. L'iniquité entre les genres s'appuie largement sur les normes, les relations et les rôles genrés, définis dans l'encadré 1. Par exemple, les rôles genrés expliquent pourquoi les femmes médecins qui ont des enfants consacrent 100,2 minutes de plus par jour aux tâches ménagères et aux soins aux enfants comparativement à leurs collègues masculins ${ }^{17}$. Cela ajoute à la difficulté des femmes médecins qui ont des enfants d'avancer professionnellement. Les normes genrées expliquent pourquoi, comparativement aux individus des autres genres, les hommes sont plus nombreux à se faire offrir des postes de responsabilité et à recevoir des lettres de recommandation plus affirmatives. En outre, les relations genrées expliquent pourquoi les hommes subissent moins de conséquences d'incivilités ou de gestes de harcèlement en milieu 
de travail. Une récente étude observationnelle sur la culture qui prévaut dans les blocs opératoires a analysé la prévalence et les prédicteurs d'une exposition à des comportements perturbateurs dans les blocs opératoires ${ }^{18}$. Un comportement perturbateur se définissait par un ensemble de gestes inacceptables en milieu de travail, tels que incivilité, intimidation et harcèlement. Un autre terme utilisé est « comportement interpersonnel (c.-à-d., visant autrui ou posé en présence d'autrui) et désigne un comportement menaçant pour la personne visée ou les témoins et enfreignant les règles de conduite respectueuse normalement attendue d'une personne ${ }^{18}$. L'étude a constaté que les femmes médecins signalent davantage d'expositions à des comportements perturbateurs et ressentent significativement moins de confiance et de contrôle quant à leur capacité de poser des gestes pour s'opposer à l'incivilité dans leur établissement hospitalier ou universitaire ${ }^{18}$. Le harcèlement genré et sexuel peut être associé à des milieux où prévaut l'iniquité aux chapitres de la rémunération et des possibilités d'avancement et de promotion ${ }^{19,20}$. Les comportements perturbateurs et le harcèlement explicite perdurent probablement dans nos établissements de santé parce que les contrevenants sont considérés comme essentiels à l'organisation en raison de leur stature, de leur leadership, de leur productivité ou de leur réputation ${ }^{19,20}$ et sont rarement réprimandés pour leurs gestes, ce qui amplifie les iniquités entre les genres.

\section{Pourquoi faut-il plus d'équité en médecine?}

Promouvoir l'équité entre les genres en médecine est une question de justice et de droits. Le fait d'avoir plus de femmes médecins et plus de femmes au sommet de la hiérarchie décisionnelle pour les politiques en santé semble aussi propice à une optimisation de la qualité des soins. Selon de grandes études observationnelles rigoureuses, comparativement aux patients d'hommes médecins ${ }^{22}$, les patients de femmes médecins se sentent mieux pris en charge pour leur diabète ${ }^{21}$ et ils présentent des taux significativement moindres de mortalité22-24, de réhospitalisations ${ }^{22}$ et de consultations aux services des urgences ${ }^{25}$. Une étude a expliqué ce phénomène comme suit : les femmes passent plus de temps avec leurs patients, leur approche est plus axée sur les patients et les soins qu'elles prodiguent se basent davantage sur les données probantes ${ }^{22}$. Deux récents articles d'opinion mentionnent des recherches selon lesquelles, la représentation des femmes dans les conseils d'administration, des hôpitaux, entre autres, donne lieu à des décisions plus "socialement orientées » et à moins de corruption ${ }^{26,27}$. Faute d'équité entre les genres, nous risquons de passer à côté de solutions créatives à des problèmes de santé complexes ${ }^{26}$ et surtout, nous privons les patients de soins optimau ${ }^{20}$.

\section{Comment atteindre l'équité entre les genres en médecine?}

Même si, comme solution, il est tentant de fournir aux gens une formation sur la diversité et les préjugés inconscients et de clarifier les limites des comportements non professionnels pour venir à bout de l'iniquité entre les genres ${ }^{28}$, ces interventions ne sont qu'un petit pas vers une plus grande sensibilisation au problème. De plus, leur effet est de courte durée et elles peuvent même être nuisibles si la responsabilité de l'iniquité est mal attribuée et qu'aucune mesure systémique n'est mise en place pour la contrer.

Il n'y a pas de remède miracle contre l'iniquité entre les genres. Il faut des interventions multidimensionnelles alliant des mesures structurelles et individuelles (résumées dans l'encadré 3) pour obtenir des changements durables et déterminants ${ }^{29-38}$. Selon Hui et ses collègues ${ }^{39}$, des préjugés sexistes implicites prévalent tout au long de la formation et de la pratique médicales. Les solutions doivent donc commencer par une reconnaissance de la nature systémique du problème. Les solutions doivent également être holistiques et appuyées par les organisations professionnelles, y compris aux échelons national (p. ex., AMC, Association des facultés de médecine du Canada, Collège royal des médecins et chirurgiens du Canada et Collège des médecins de famille du Canada), provincial (p. ex., associations médicales des provinces et des territoires) et local (p. ex., direction des hôpitaux, des cliniques, des cabinets et des facultés).

\section{Mesurer les iniquités entre les genres}

Documenter les données sur les iniquités entre les genres et autres données intersectionnelles et les rendre publiques permettrait de faire mieux connaître les iniquités, comme le montre une étude de cas de 2015 sur l'équité entre les genres chez les conférenciers invités dans les congrès médicaux ${ }^{40}$ qui a mis le problème en lumière; alliées à une responsabilité publique, les observations ont suscité des changements et permis d'en suivre les répercussions. Le travail qui consiste à mesurer les iniquités entre les genres, à les rendre publiques et à inciter à des changements pour plus d'équité doit être reconnu et rémunéré en conséquence.

\section{À la défense des changements comportementaux et systémiques}

Pour faire évoluer les comportements et les systèmes, l'exemple doit venir de haut ${ }^{41}$. C'est ce qu'a démontré une étude de cas sur une intervention qui mesurait l'effet d'un changement structurel descendant dans les facultés de sciences, de technologie, de génie, de mathématiques et de médecine à l'Université de l'état de l'Oregon. L'intervention a pris la forme d'un séminaire de réflexion approfondie sur les systèmes d'oppression et le pouvoir dans la haute hiérarchie universitaire. Les conclusions ont mené à la mise en place de plans d'action et de politiques qui orientaient les facultés vers plus d'équité et de justice, avec des paramètres mesurables. De plus, lorsque les cadres facultaires supérieurs ont des comportements pro-équité, cela favorise un climat plus inclusif et positif au sein des facultés. Les responsables des sociétés professionnelles et des établissements universitaires et médicaux devraient se porter garants 
Encadré 3 : Solutions pour lutter contre l'iniquité dans les milieux universitaires en médecine*

Quantification des iniquités entre les genres sur les plans du financement, des publications, des promotions et de la rémunération

- Faire connaître les statistiques liées aux genres

- Publier un rapport annuel sur les retombées des efforts déployés pour plus d'équité entre les genres (rédigé et publié pour plus de responsabilisation)

\section{Changement comportemental ou systémique}

- Reconnaissance de la nature systémique de l'iniquité entre les genres et de la nécessité d'apporter des solutions systémiques dans les organisations

- Principes d'équité démontrés par l'exemple de la part des responsables des établissements professionnels (universités et milieux de soins)

- Inclusion des principes fondamentaux de l'équité, diversité, inclusion, respect mutuel, collégialité et professionnalisme, dans toutes les politiques organisationnelles

- Énoncé clair des objectifs pour venir à bout des iniquités; description précise des mesures et des plans à cet effet

- Marches à suivre et politiques ouvertes et transparentes pour protéger les lanceurs d'alertes

- Correctifs appropriés et fondés sur des données probantes pour les contrevenants en présence d'une cause identifiable (p. ex., épuisement professionnel ou problème de santé mentale)

- Langage inclusif (épicène) lors du recrutement, de l'embauche et des évaluations en vue de subventions ou de financement

- Utilisation de quotas inversés

- Comités recruteurs reflétant la diversité de la profession ou de la population élargie et sensibilité du comité aux préjugés sexistes dans les lettres de recommandation

- Formation sur les préjugés sexistes et défense active de l'équité entre les genres

- Séminaires de formation et programmes basés sur la théorie des systèmes d'oppression avec des spécialistes de la question

- Approche proportionnelle pour les subventions de recherche

\section{Flexibilité professionnelle}

- Planification intégrée de la conciliation carrière-vie personnelle, mesures d'aide à l'élaboration de plans personnalisés pour l'atteinte d'objectifs professionnels et personnels, et système de banque de temps

- Politiques flexibles, y compris politiques de conciliation travail-famille, congés parentaux et flexibilité professionnelle

- Plans de congés parentaux non genrés

- Abrègement des journées de travail

- Politiques interdisant l'assignation de tâches ou l'envoi de courriels les soirs et les fins de semaine

\section{Visibilité, reconnaissance et} représentativité accrues

- Planification du développement professionnel

- Programme de leadership

- Présentation de modèles favorisant la compatibilité des identités et le sentiment d'appartenance

- Campagne dans les médias sociaux

Mises en place d'initiatives de développement, de mentorat et de parrainage

- Plan d'orientation professionnelle

- Programme de révision des curriculum vitae

- Programme de mentorat par les pairs

- Programme de parrainage

\section{Soutien financier}

- Soutien financier pour les responsabilités parentales et de proche aidant

- Loterie des subventions de recherche

*On recommande des solutions qui allient plusieurs des éléments énumérés ici. des principes d'équité. Toutefois, même si les autorités en place doivent donner l'exemple, cela ne suffit pas. Les principes de base de l'équité, la diversité, l'inclusion, le respect mutuel, la collégialité et le professionnalisme, doivent être enchâssés dans chaque politique, programme et marche à suivre, depuis les études de premier cycle jusqu'aux cycles supérieurs et jusqu'à la pratique clinique et au leadership professionnel. Par l'entremise de leurs conseils d'établissements et de leur haute direction, les organisations doivent décrire clairement leurs objectifs pour lutter contre les iniquités et expliquer comment elles entendent les atteindre. Elles doivent aussi être responsables. De plus, les alliés et lanceurs d'alertes, tous genres confondus, qui signalent des comportements non professionnels doivent bénéficier de soutien et de protection grâce à des mécanismes ouverts et transparents qui leur permettent de s'exprimer ${ }^{18}$, et le comportement des contrevenants doit être corrigé conformément aux pratiques optimales et aux données probantes, selon les circonstances.

\section{Sélection et embauche}

L'évolution d'une organisation médicale vers une culture d'équité devrait idéalement commencer dès les inscriptions à la faculté de médecine et se poursuivre à toutes les étapes du développement professionnel. Comme les préjugés sexistes implicites sont fréquents, et par définition, peu reconnus ${ }^{42,43}$, la formation à cet égard est essentielle pour les responsables de la sélection des personnes candidates, même si en l'absence de mesures claires pour modifier les comportements, de telles formations pourraient ne pas suffire. Des marches à suivre et des politiques ouvertes et transparentes favorisant l'équité en matière d'embauche des candidates et candidats universitaires et cliniques ${ }^{11}$ et des procédures de recrutement ouvertes (incluant l'affichage des postes) devraient comporter des critères liés à l'équité. Par exemple, quiconque est admissible devrait être encouragé à poser sa candidature et des stratégies actives devraient être appliquées pour accroître la diversité des candidatures. Le libellé des affichages de postes devrait être neutre (épicène) et ne pas favoriser implicitement un 
genre au détriment des autres; il existe des guides en ligne de rédaction épicène ${ }^{44}$. Certains procédés spécifiques permettent de réduire les préjugés sexistes implicites, comme le caractère à l'insu, et favorisent une diversité de candidatures ${ }^{45}$, mais l'utilisation d'un langage non genré (épicène) ne s'est pas révélé efficace pour réduire les préjugés sexistes dans les processus de demande de subventions ${ }^{46}$.

En plus de recevoir une formation sur les préjugés, les comités de recrutement devraient eux-mêmes refléter la diversité de la population et augmenter ainsi la probabilité que les candidatures de la diversité soit traitées de manière équitable durant le processus de sélection. Disposer d'un agent de recrutement qui a suivi une formation pour siéger aux comités de recrutement peut aussi témoigner d'un engagement à l'endroit de l'équité et de l'inclusion et aider les comités de recrutement dans leurs efforts pour éviter les préjugés inconscients et involontaires. Tous les comités devraient exiger une formation sur l'équité, la diversité et l'inclusivité pour assurer la sélection des meilleures candida-

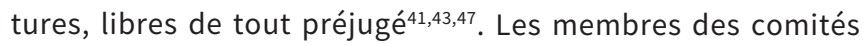
doivent aussi être sensibles aux préjugés sexistes dans les lettres de recommandation (p. ex., accent placé sur les relations c. réalisations des candidates ${ }^{48}$ ). Des paramètres de mesure des capacités non traditionnels, comme les retombées du travail des personnes candidates, plutôt que le nombre de leurs publications, devraient attirer l'attention pendant les entrevues, y compris toute expertise nouvelle en matière d'équité entre les genres. Étant donné que la productivité des candidates peut avoir été affectée par le temps requis pour élever une famille ou accomplir des tâches domestiques non rémunérées, il importe d'appliquer divers paramètres pour évaluer la productivité et l'efficience. Si on ne tient compte que du nombre de publications, de présentations et de subventions obtenues, un biais de sélection en faveur des candidats de sexe masculin s'exercera. Prioriser d'autres critères, tels que les évaluations des étudiants, des patients et des collègues permet un classement plus équitable. L'utilisation de quotas inversés ${ }^{49}$ (p. ex., que seulement $50 \%$ du leadership soit exercé par des hommes) devrait être encouragée pour promouvoir les nominations au mérite et neutraliser le privilège de masculinité ${ }^{50}$.

\section{Soutien à la carrière des femmes}

Le soutien actuel à la carrière des femmes est minime et inadéquat. Pour attirer et retenir des candidates de talent, les universités devront se doter de politiques capables d'assurer un parcours professionnel flexible dans un environnement propice qui remettra en question la norme du «travailleur idéal ${ }^{51,52}$. Des initiatives favorisant la réussite des équipes, comportant des mesures de conciliation travail-vie personnelle et travail-travail pourraient inclure, une planification professionnelle-familiale intégrée, une formation pour élaborer un plan personnalisé permettant de réaliser ses objectifs professionnels et personnels, et des systèmes de banques de temps ${ }^{53}$. Ces derniers permettent de mesurer le travail non reconnu, comme l'enseignement, les services et activités cli- niques, et le reconnaissent par des gratifications pratiques sous forme de services de soutien axés sur les objectifs professionnels et personnels, en allégeant les contraintes de temps et en encourageant la réussite professionnelle ${ }^{54}$. Les politiques de congés parentaux et de conciliation travail-famille incluent des plans de remplacement de revenus pour offrir plus de ressources aux familles ${ }^{55-57}$. Des données probantes non spécifiques aux médecins indiquent que jusqu'à 6 mois de congé parental payé, permettent d'accroître la participation des femmes au marché de l'emploi et réduisent les inégalités salariales ${ }^{58}$. Ajoutons que rendre le congé parental accessible aux hommes et aux femmes est crucial pour leur offrir des chances égales au plan économique. Deux études qui ont analysé le congé payé en Californie ont constaté qu'il augmentait 10\%-17\% le nombre habituel d'heures travaillées par les mères d'enfants agés 1-3 ans. Les études ont aussi démontré un lien entre cette politique et la probabilité de travail et d'emploi chez les mères, 9-12 mois après l'accouchement ${ }^{59,60}$. De plus, 1-5 ans après l'accouchement, le revenu maternel augmente quand les mères ont accès à un congé de maternité payé de durée modérée ${ }^{61,62}$. Le soutien financier à l'intention des membres facultaires ayant des responsabilités familiales permettrait la fidélisation du personnel et favoriserait l'accès à des promotions ${ }^{63}$.

Pour les groupes minoritaires, on déplore un manque de femmes modèles et mentores dans les milieux universitaires en médecine. Pour augmenter la visibilité des femmes issues de la diversité dans tous les domaines de la médecine universitaire, des plans d'orientation de carrière pourraient améliorer leur confiance en leur capacité de faire valoir leurs compétences, d'obtenir de l'avancement, de parfaire leur formation ou de diversifier leurs possibilités d'emploi64. Le mentorat par des pairs de même sexe favoriserait la reconnaissance et la représentativité ${ }^{65-69}$. Les femmes mentores et modèles en milieu universitaire ont une importante influence sur l'orientation professionnelle, les choix de carrière, la productivité en recherche et le développement personnel ${ }^{70,71}$. En raison du manque de femmes aux échelons supérieurs de la hiérarchie médicale en milieu universitaire, il n'y a pas toujours de femmes pour jouer le rôle de mentores. Et à lui seul, le mentorat ne suffira peut-être pas à stimuler l'avancement professionnel des femmes. Ce qui pourrait être plus efficace, est le parrainage, soit un effort délibéré de la part des autorités en place pour aider plus efficacement l'avancement professionnel des femmes ${ }^{72}$. Le parrainage cible directement l'avancement professionnel et s'inscrit dans un réseau établi et un cercle d'influence significatif sur le plan des processus décisionnels ou des structures afin d'offrir des possibilités professionnelles uniques aux jeunes membres facultaires. Dans le contexte actuel, où le manque de leaders féminins se fait cruellement sentir pour exercer ce pouvoir dans les milieux universitaires en médecine, les hommes doivent être évalués en fonction de leur capacité d'offrir un tel parrainage aux femmes ${ }^{73}$.

Des approches organisationnelles s'imposent, avec des plans d'action et des paramètres mesurables et publiquement 
Encadré 4 : Charte du réseau Athena Scientific Women's Academic Network : Exemple de solution multidimensionnelles pour lutter contre l'iniquité entre les genres en milieu universitaire

La charte du réseau Athena Scientific Women's Academic Network (SWAN) encourage et enjoint les universités du Royaume-Uni (R.-U.) à favoriser la carrière des femmes en sciences, technologie, génie, mathématiques et médecine ${ }^{74}$. En tant que programme primé, le réseau SWAN d'Athena promeut l'avancement des femmes vers des postes de direction en éliminant les obstacles, en veillant à l'égalité de la rémunération et en facilitant le soutien par le biais de mesures à tous les échelons des établissements. Des comités de révision par des pairs évaluent les mises en candidatures, formulent des suggestions relatives aux subventions en plus de fournir aux candidates des commentaires constructifs. Les catégories d'interventions incluent auto-évaluation et surveillance; principaux moments décisifs professionnels; contrats permanents et à long terme, y compris sécurité d'emploi pour le personnel en milieu universitaire et scientifique; développement professionnel; promotion de politiques de travail flexibles, y compris gestion des congés professionnels; amélioration de la culture des organisations en lien avec l'équité entre les genres; et combinaison de plans d'action complexes et spécifiques aux contextes et d'organisation à l'échelle des systèmes.

La charte du réseau SWAN d'Athena est largement utilisée au R.-U comme outil pour lutter contre les iniquités entre les genres dans les établissements d'enseignement supérieur. Les femmes des échelons hiérarchiques supérieurs sont plus susceptibles d'être satisfaites de leurs évaluations de rendement et de développement, de connaître les critères et les marches suivre afférentes aux promotions, d'avoir été encouragées à poser leur candidature pour des promotions, de croire qu'il existe des pratiques de travail flexibles, d'être plus optimistes vis-à-vis des possibilités d'avancement professionnel et d'avoir accès à des plans de mentorat ${ }^{74}$. Des données ont permis de relier la charte à un degré supérieur d'engagement de la part des femmes ${ }^{74}$. Les femmes blanches de classe moyenne sont les principales bénéficiaires du réseau SWAN d'Athena. Ce réseau et d'autres initiatives similaires doivent intégrer l'intersectionnalité et les effets du recoupement entre la race et d'autres identités sociales (y compris de genre) pour les femmes en sciences, technologie, génie, mathématiques et médecine. D'autres pays ont appliqué des programmes similaires adaptés à leur situation, comme le projet Science in Australia Gender Equity (SAGE) en Australie $^{75}$ et la charte Dimensions au Canada ${ }^{76}$.

accessibles si l'on veut promouvoir des pratiques optimales dans la communauté élargie (encadré 4) ${ }^{77,78}$.

\section{Conclusion}

L'équité entre les genres en médecine deviendra réalité quand la culture aura changé à chaque échelon du système ${ }^{79}$. Si l'équité entre les genres est vraiment valorisée, il faudra de solides recherches sur les facteurs qui prédisposent à l'iniquité et sur les solutions potentielles pour les aplanir efficacement. Certaines des auteures travaillent actuellement avec des collègues de 7 pays pour mesurer l'efficacité de dif- férents types d'intervention dans la lutte pour l'équité entre les genres, avec l'appui des Instituts de recherche en santé du Canada $^{80}$. Mais plusieurs solutions fondées sur des données probantes peuvent d'ores et déjà être adoptées, il n'y a pas d'excuses pour ne pas travailler à la modification du climat et de l'environnement de travail en médecine afin qu'ils soient plus propices à la diversité. La médecine se doit d'être professionnelle et collégiale; elle doit promouvoir le respect mutuel et faciliter la contribution et la réalisation du plein potentiel des individus, sans égard au genre, à la race, à l'ethnicité, à la religion et à la nationalité, au bénéfice des patients. L'équité n'existera que lorsque chacun, indépendamment de son genre ou autre particularité, bénéficiera de l'équité sur les plans de la rémunération, des promotions et autres aspects. Le temps n'est pas mieux choisi pour mettre de l'avant des politiques qui appuient l'équité en médecine.

\section{Références}

1. Gender and health. Geneva: World Health Organization. Accessible ici : www. who.int/health-topics/gender\#tab=tab_1 (consulté le 17 nov. 2020).

2. Glauser W. Rise of women in medicine not matched by leadership roles. CMAJ 2018;190:E479-80.

3. Jena AB, Khullar D, Ho O, et al. Sex differences in academic rank in US medical schools in 2014. JAMA 2015;314:1149-58.

4. Canadian Medical Association; Federation of Medical Women of Canada. Addressing gender equity and diversity in Canada's medical profession: a review. Ottawa: Canadian Medical Association.

5. Robb N. Canada has its first female dean -170 years after first medical school opened. CMAJ 1999;160:1042.

6. Yu PT, Parsa PV, Hassanein O, et al. Minorities struggle to advance in academic medicine: a 12-y review of diversity at the highest levels of America's teaching institutions. J Surg Res 2013;182:212-8.

7. Witteman HO, Hendricks M, Straus S, et al. Gender bias in CIHR Foundation grant awarding. Lancet 2019;394:e41-2.

8. Witteman HO, Hendricks M, Straus S, et al. Are gender gaps due to evaluations of the applicant or the science? A natural experiment at a national funding agency. Lancet 2019;393:531-40.

9. Merman E, Pincus D, Bell C, et al. Differences in clinical practice guideline authorship by gender. Lancet 2018;392:1626-8.

10. Buell D, Hemmelgarn BR, Straus SE. Proportion of women presenters at medical grand rounds at major academic centres in Canada: a retrospective observational study. BMJ Open 2018;8:e019796.

11. Cohen M, Kiran T. Closing the gender pay gap in Canadian medicine. CMAJ 2020;192:E1011-7.

12. Boesveld S. What's driving the gender pay gap in medicine? CMAJ 2020;192:E19-20.

13. Trix F, Psenka C. Exploring the color of glass: letters of recommendation for female and male medical faculty. Discourse Soc 2003;14:191-220.

14. Kang SK, Kaplan S. Working toward gender diversity and inclusion in medicine: myths and solutions. Lancet 2019;393:579-86.

15. Glauser W. How female physicians are supporting each other in addressing professional inequities. CMAJ 2019;191:E485-6.

16. Pololi LH, Civian JT, Brennan RT, et al. Experiencing the culture of academic medicine: gender matters, a national study. J Gen Intern Med 2013;28:201-7.

17. Ly DP, Jena AB. Sex differences in time spent on household activities and care of children among US physicians, 2003-2016. Mayo Clin Proc 2018;93:1484-7.

18. Villafranca A, Hiebert B, Hamlin C, et al. Prevalence and predictors of exposure to disruptive behaviour in the operating room. Can J Anaesth 2019;66:781-94.

19. Pattani R, Ginsburg S, Mascarenhas Johnson A, et al. Organizational factors contributing to incivility at an academic medical center and systems-based solutions: a qualitative study. Acad Med 2018;93:1569-75.

20. Choo EK, Byington CL, Johnson N-L, et al. From \#MeToo to \#TimesUp in health care: can a culture of accountability end inequity and harassment? Lancet 2019;393:499-502.

21. Berthold HK, Gouni-Berthold I, Bestehorn KP, et al. Physician gender is associated with the quality of type 2 diabetes care. J Intern Med 2008;264:340-50.

22. Tsugawa Y, Jena AB, Figueroa JF, et al. Comparison of hospital mortality and readmission rates for medicare patients treated by male vs female physicians. JAMA Intern Med 2017;177:206-13. 
23. Wallis CJ, Ravi B, Coburn N, et al. Comparison of postoperative outcomes among patients treated by male and female surgeons: a population based matched cohort study. BMJ 2017;359:j4366.

24. Greenwood BN, Carnahan S, Huang L. Patient-physician gender concordance and increased mortality among female heart attack patients. Proc Natl Acad Sci U S A 2018;115:8569-74.

25. Dahrouge S, Seale E, Hogg W, et al. A comprehensive assessment of family physician gender and quality of care: a cross-sectional analysis in Ontario, Canada. Med Care 2016;54:277-86

26. Fine C, Sojo V. Women's value: beyond the business case for diversity and inclusion. Lancet 2019;393:515-6.

27. Downs JA, Reif LK, Hokororo A, et al. Increasing women in leadership in global health. Acad Med 2014;89:1103-7.

28. Tricco AC, Rios P, Zarin W, et al. Prevention and management of unprofessional behaviour among adults in the workplace: a scoping review. PLoS One 2018;13:e0201187.

29. Richman RC, Morahan PS, Cohen DW, et al. Advancing women and closing the leadership gap: the Executive Leadership in Academic Medicine (ELAM) program experience. J Womens Health Gend Based Med 2001;10:271-7.

30. McDade SA, Richman RC, Jackson GB, et al. Effects of participation in the Executive Leadership in Academic Medicine (ELAM) program on women faculty's perceived leadership capabilities. Acad Med 2004;79:302-9.

31. Dannels SA, Yamagata H, McDade SA, et al. Evaluating a leadership program: a comparative, longitudinal study to assess the impact of the Executive Leadership in Academic Medicine (ELAM) program for women. Acad Med 2008;83:488-95.

32. Bauman MD, Howell LP, Villablanca AC. The women in medicine and health science program: an innovative initiative to support female faculty at the University of California Davis School of Medicine. Acad Med 2014;89:1462-6.

33. Levine RB, González-Fernández M, Bodurtha J, et al. Implementation and evaluation of the Johns Hopkins University School of Medicine leadership program for women faculty. J Womens Health (Larchmt) 2015;24:360-6.

34. Ovseiko PV, Chapple A, Edmunds LD, et al. Advancing gender equality through the Athena SWAN Charter for Women in Science: an exploratory study of women's and men's perceptions. Health Res Policy Syst 2017;15:12.

35. Kalpazidou Schmidt E, Ovseiko PV, Henderson LR, et al. Understanding the Athena SWAN award scheme for gender equality as a complex social intervention in a complex system: analysis of Silver award action plans in a comparative European perspective. Health Res Policy Syst 2020;18:19.

36. Rosser SV, Barnard S, Carnes M, et al. Athena SWAN and ADVANCE: effectiveness and lessons learned. Lancet 2019;393:604-8.

37. Dannels SA, McLaughlin JM, Gleason KA, et al. Dental school deans' perceptions of the organizational culture and impact of the ELAM program on the culture and advancement of women faculty. J Dent Educ 2009;73:676-88.

38. McDade SA, Nooks KA, King PJ, et al. A window into the culture of leadership within higher education through the leadership definitions of women faculty: a case study of ELAM women faculty alumnae. NASPA Journal About Women in Higher Education 2008;1:76-104.

39. Hui K, Sukhera J, Vigod S, et al. Recognizing and addressing implicit gender bias in medicine. CMAJ 2020;192;E1269-70.

40. Casadevall A. Achieving speaker gender equity at the American Society for Microbiology general meeting. MBio 2015;6:e01146.

41. Shaw SM, Bothwell M, Furman K, et al. Advancing women in STEM: institutional transformation. Lancet 2019;393:e17-8.

42. Girod S, Fassiotto M, Grewal D, et al. Reducing implicit gender leadership bias in academic medicine with an educational intervention. Acad Med 2016;91:1143-50.

43. Carnes M, Bartels CM, Kaatz A, et al. Why is John more likely to become department chair than Jennifer? Trans Am Clin Climatol Assoc 2015;126:197-214.

44. Gender decoder for job ads. Accessible ici : http://gender-decoder.katmatfield. com/ (consulté le 19 nov. 2020).

45. Alvarez SNE, Jagsi R, Abbuhl SB, et al. Promoting gender equity in grant making: What can a funder do? Lancet 2019;393:e9-11.

46. Tricco AC, Thomas SM, Antony J, et al. Strategies to prevent or reduce gender bias in peer review of research grants: a rapid scoping review. PLoS One 2017;12:e0169718.

47. Carr PL, Gunn C, Raj A, et al. Recruitment, promotion, and retention of women in academic medicine: how institutions are addressing gender disparities. Womens Health Issues 2017;27:374-81.

48. Grimm LJ, Redmond RA, Campbell JC, et al. Gender and racial bias in radiology residency letters of recommendation. J Am Coll Radiol 2020;17:64-71.

49. Coe IR, Wiley R, Bekker L-G. Organisational best practices towards gender equality in science and medicine. Lancet 2019;393:587-93.

50. Murray R. Quotas for men: reframing gender quotas as a means of improving representation for all. Am Polit Sci Rev 2014;108:520-32.

51. Valantine $\mathrm{H}$, Sandborg $\mathrm{Cl}$. Changing the culture of academic medicine to eliminate the gender leadership gap: 50/50 by 2020. Acad Med 2013;88:1411-3.
52. Howell LP, Beckett LA, Villablanca AC. Ideal worker and academic professional identity: perspectives from a career flexibility educational intervention. $\mathrm{Am} \mathrm{J}$ Med 2017;130:1117-25.

53. Fassiotto M, Simard C, Sandborg C, et al. An integrated career coaching and time-banking system promoting flexibility, wellness, and success: a pilot program at Stanford University School of Medicine. Acad Med 2018;93:881-7.

54. Fassiotto M, Maldonado YA. A time banking system to support workplace flexibility Stanford Medicine, WellMD. Accessible ici : https://wellmd.stanford.edu/content/ dam/sm/wellmd/documents/Time-banking-system.pdf (consulté le 19 nov. 2020).

55. Villablanca AC, Beckett L, Nettiksimmons J, et al. Career flexibility and familyfriendly policies: an $\mathrm{NIH}$-funded study to enhance women's careers in biomedical sciences. J Womens Health (Larchmt) 2011;20:1485-96.

56. Villablanca AC, Li Y, Beckett LA, et al. Evaluating a medical school's climate for women's success: outcomes for faculty recruitment, retention, and promotion. J Womens Health (Larchmt) 2017;26:530-9.

57. Patnaik A. Reserving time for Daddy: the consequences of fathers' quotas. $J$ Labor Econ 2019;37:1009-59.

58. Brugiavini A, Pasini G, Trevisan E. The direct impact of maternity benefits on leave taking: evidence from complete fertility histories. Adv Life Course Res 2013;18:46-67.

59. Baum CL II, Ruhm CJ. The effects of paid family leave in California on labor market outcomes. J Policy Anal Manage 2016;35:333-56.

60. Rossin-Slater M, Ruhm CJ, Waldfogel J. The effects of California's paid family leave program on mothers' leave-taking and subsequent labor market outcomes. J Policy Anal Manage 2013;32:224-45.

61. Waldfogel J. The family gap for young women in the United States and Britain Can maternity leave make a difference? J Labor Econ 1998;16:505-45.

62. Rasmussen AW. Increasing the length of parents' birth-related leave: the effect on children's long-term educational outcomes. Labour Econ 2010;17:91-100.

63. Jagsi R, Butterton JR, Starr R, et al. A targeted intervention for the career develop ment of women in academic medicine. Arch Intern Med 2007;167:343-5.

64. Gottlieb AS, Travis EL. Rationale and models for career advancement sponsor ship in academic medicine: the time is here; the time is now. Acad Med 2018;93:1620-3.

65. Von Feldt JM, Bristol M, Sonnad S, et al. The brief CV review session: one component of a mosaic of mentorship for women in academic medicine. $\mathrm{J} \mathrm{Nat}$ Med Assoc 2009;101:873-80.

66. Gardiner M, Tiggemann M, Kearns H, et al. Show me the money! An empirical analysis of mentoring outcomes for women in academia. High Educ Res Dev 2007;26:425-42.

67. Files JA, Blair JE, Mayer AP, et al. Facilitated peer mentorship: a pilot program for academic advancement of female medical faculty. J Womens Health (Larchmt) 2008;17:1009-15

68. Varkey $\mathrm{P}$, Jatoi A, Williams A, et al. The positive impact of a facilitated peer mentoring program on academic skills of women faculty. BMC Med Educ 2012;12:14.

69. Dutta R, Hawkes SL, Kuipers E, et al. One year outcomes of a mentoring scheme for female academics: a pilot study at the Institute of Psychiatry, King's College London. BMC Med Educ 2011;11:13.

70. Rosenthal L, Levy SR, London B, et al. In pursuit of the MD: the impact of role models, identity compatibility, and belonging among undergraduate women. Sex Roles 2013;68:464-73.

71. Butkus R, Serchen J, Moyer DV, et al. Achieving gender equity in physician compensation and career advancement: a position paper of the American College of Physicians. Ann Intern Med 2018;168:721-3.

72. Spector ND, Asante PA, Marcelin JR, et al. Women in pediatrics: progress, barriers, and opportunities for equity, diversity, and inclusion. Pediatrics 2019;144:e20192149.

73. Bates C, Gordon L, Travis E, et al. Striving for gender equity in academic medicine careers: a call to action. Acad Med 2016;91:1050-2.

74. Graves A, Rowell A, Hunsicker E. An impact evaluation of the Athena SWAN Charter. Newcastle upon Tyne (UK): Ortus Economic Research; Loughborough (UK): Loughborough University; 2019.

75. SAGE: Science in Australia Gender Equity [main page]. Accessible ici www.sciencegenderequity.org.au (consulté le 17 nov. 2020).

76. Equity, diversity and inclusion. Ottawa: Natural Sciences and Engineering Research Council. Accessible ici : www.nserc-crsng.gc.ca/NSERC-CRSNG/EDI -EDI/Dimensions-Charter_Dimensions-Charte_eng.asp (consulté le 30 nov. 2020).

77. Jagsi R, Spector ND. Leading by design: lessons for the future from 25 years of the Executive Leadership in Academic Medicine (ELAM) program for women. Acad Med 2020;95:1479-82.

78. Plank-Bazinet JL, Heggeness ML, Lund PK, et al. Women's careers in biomedical sciences: implications for the economy, scientific discovery, and women's health. J Womens Health (Larchmt) 2017;26:525-9.

79. Pritlove C, Juando-Prats C, Ala-Leppilampi K, et al. The good, the bad, and the ugly of implicit bias. Lancet 2019;393:502-4. 


\section{Intérêts concurrents : Aucun déclaré.}

Cet article a été commandé et a été révisé par les pairs.

Affiliations : Programme d'application des connaissances (Tricco, Peer, Straus), Institut du savoir Li Ka Shing, Hôpital St. Michael, Unity Health Toronto; Division d'épidémiologie et Institut de gestion, d'évaluation et de politiques de santé (Tricco), École de santé publique Dalla Lana, Université de Toronto, Toronto, Ont.; École de sociologie et d'anthropologie (Bourgeault), Université d'Ottawa, Ottawa, Ont.; Département de médecine familiale (Moore), Université McMaster, Hamilton, Ont.; Département de médecine familiale et communautaire (Grunfeld) et Département de médecine gériatrique (Straus), Université de Toronto, Toronto, Ont.

Collaborateurs : Andrea Tricco a rédigé la première version du manuscrit. Toutes les auteures ont révisé de façon critique le contenu intellectuel important du manuscrit; elles ont donné leur approbation finale pour la version destinée à être publiée et assument l'entière responsabilité de tous les aspects du travail.

Financement : Andrea Tricco est titulaire d'une chaire de recherche du Canada en synthèse des connaissances de niveau 2. Ivy
Bourgeault est subventionnée par la Chaire de recherche sur le genre, l'égalité et l'inclusion dans les milieux professionnels et par Condition féminine Canada, ON 16255, Renforcement du pouvoir des dirigeantes dans le secteur de la santé. Eva Grunfeld est bénéficiaire d'une bourse de clinicienne-chercheuse de l'Institut ontarien de recherche sur le cancer et détentrice de la Chaire de recherche Giblon en médecine familiale. Sharon Straus est titulaire d'une chaire de recherche du Canada sur l'application des connaissances de niveau 1.

Propriété intellectuelle du contenu : Il s'agit d'un article en libre accès distribué conformément aux modalités de la licence Creative Commons Attributions (CC BY-NC-ND 4.0), qui permet l'utilisation, la diffusion et la reproduction dans tout médium à la condition que la publication originale soit adéquatement citée, que l'utilisation se fasse à des fins non commerciales (c.-à-d. recherche ou formation) et qu'aucune modification ni adaptation n'y soit apportée. Voir : https:// creativecommons.org/licenses/by-nc-nd/4.0/deed.fr.

Remerciements : Les auteures tiennent à remercier Shazia Siddiqui qui a aidé au formatage de l'article.

Correspondance : Andrea Tricco, Andrea.Tricco@unityhealth.to 